\title{
Anomalous $g$-Factors for Charged Leptons in a Fractional Coarse-Grained Approach
}

\author{
J. Weberszpil ${ }^{1}$ and J. A. Helayël-Neto ${ }^{2}$ \\ ${ }^{1}$ Universidade Federal Rural do Rio de Janeiro, UFRRJ-IM/DTL, Avenida Governador Roberto Silveira s/n, \\ 695014 Nova Iguaçú, RJ, Brazil \\ ${ }^{2}$ Centro Brasileiro de Pesquisas Físicas-CBPF, Rua Dr. Xavier Sigaud 150, 22290-180 Rio de Janeiro, RJ, Brazil
}

Correspondence should be addressed to J. Weberszpil; josewebe@gmail.com

Received 20 March 2014; Accepted 4 May 2014; Published 25 May 2014

Academic Editor: Kingman Cheung

Copyright (c) 2014 J. Weberszpil and J. A. Helayël-Neto. This is an open access article distributed under the Creative Commons Attribution License, which permits unrestricted use, distribution, and reproduction in any medium, provided the original work is properly cited. The publication of this article was funded by SCOAP ${ }^{3}$.

We here propose to extend the concept of helicity to include it in a fractional scenario and we write down the left- and the righthanded Weyl equations from first principles in this extended framework. Next, by coupling the different fractional Weyl sectors by means of a mass parameter, we arrive at the fractional version of Dirac's equation which, whenever coupled to an external electromagnetic field and reduced to the nonrelativistic regime, yields a fractional Pauli-type equation. From the latter, we are able to present an explicit expression for the gyromagnetic ratio of charged fermions in terms of the fractionality parameter. We then focus our efforts to relate the coarse-grained property of space-time to fractionality and to the $(g-2)$ anomalies of the different leptonic species.

\section{Introduction}

The electron's gyromagnetic ratio, $g_{e}$, is a dimensionless parameter that appears in the spin-orbit interaction and leads to a splitting in the atomic energy levels, giving rise to the so-called fine structure. The $g_{e}$-factor appears in the Zeeman effect, by means of the electron's magnetic dipole moment, in the description of the interaction of atoms with external magnetic fields.

It is now known that the anomalous magnetic moment (AMM) of the muon is considered to be one of the most promising observables that can unveil effects of some new physics beyond the Standard Model [1]. One also claims that the muon's AMM is one of the most precisely measured quantities in particle physics, reaching a precision of $0.54 \mathrm{ppm}$ [2]. This statement is also reinforced in view of the relatively large value of the muon's mass [3]. The tau-lepton gyromagnetic ratio is not very well known experimentally and for this reason it does not provide yet a good test of the Standard Model (SM) and for physics beyond the Standard Model [3].
Nonrelativistic quantum mechanics predicts the remarkable result, $g_{e}=2$. Experimentally, the electron's $g$ ratio has been measured with high precision and the "2010 CODATA value" [4] for this parameter is $g_{e, \exp }=$ 2.00231930436153(53), which is supported by the theoretical calculations of Quantum Electrodynamics (QED), slightly different from that of Dirac's theoretical prevision in the realm of Quantum Mechanics (QM). A good review on the introduction and history of the $g$-ratios, up to 2007, may be found in [5]. For a nonperturbative approach, the reader could consult [6]. An update of the electron's and muon's $g$ ratios can be found in [3].

In this paper, we investigate the fractional coarse-grained aspects of the electron's anomalous magnetic $g$-ratio, showing the possibility of mapping its experimental value into some theoretical parameter that accounts for fractionality, so that we take the viewpoint that fractionality could be responsible for the deviation from the $g_{e}=2$ relativistic quantummechanical result. This claim highlights that our motivation to adopt the fractional calculus (FC) is more physical than 
a simple mathematically motivated extension of classical calculus. This becomes more explicit once we argue that the possible justification for the experimental difference from the theoretical Dirac's value $g_{e}=2$ is imputed to the complexity of the interactions of the electrons, taken as a pseudoparticle "dressed" by the interactions and the medium. Here, we look at a dynamical system as an open system that can interact with the environment and we argue that FC may become an important tool to study open classical and quantum systems [7].

FC is one of the possible generalizations of classical calculus. It provides a redefinition of a number of mathematical concepts and it seems very useful to deal with anomalous and frictional systems [8-13]. Several applications of FC may be found in the literature [14-17]. Presently, areas such as field theory and gravitational models demand new conceptions and approaches which might allow us to understand unexplored systems and could help in extending well-known results. Interesting problems may be related to the quantization of field theories for which new approaches have been proposed [18-21]. In connection with our work, it is worthy to mention here that a fractional Riemann-Liouville Zeeman effect, an attempt to implement gauge invariance in fractional field theories and, an angular momentum algebra proposed with the Riemann-Liouville formalism are reported in the paper [22]. Low-energy nuclear excitations have been studied in terms of a fractional symmetric rigid rotor with the purpose of calculating baryonic excitations [23].

Fractional systems are described as being dissipative [24, 25]. The use of FC is also justified here on the basis of our proposition that there exists an intimate relationship between dissipation, coarse-grained medium, and a limit energy scale for the interactions. Since we are dealing with open systems, as we already commented, particles should in fact be seen as dressed entities or pseudoparticles that exchange energy with other particles and the environment. Depending on the energy scale, a particular interaction may affect the geometry of space-time. The system composed by particles and their surroundings may be considered nonconservative due to the possible energy exchange. This energy exchange may be responsible for the resulting noninteger dimension of spacetime, giving rise then to an effective coarse-grained medium. This is quite reasonable since even standard field theory comes across a granularity in the limit of Planck scale. Also, if we consider a nonstandard geometry, such as the space-time of a cosmological black hole or the space in the surroundings of a region of pair creation, a coarse-grained view of the dynamics of open systems may become a sensible description. Here, we argue that FC allows us to describe and emulate this kind of dynamics without explicit many-body contributions, dissipation, or geometrical terms in the dynamical equations of the system. In some way, FC may yield an effective theory, with some statistical average without imposing any specific nonstandard statistics. So, FC may be the tool that could describe in a consistent way the connections between coarsegrained medium and dissipation at a certain energy scale.

It is worthy to point out here the significant work by Svozil and Zeilinger [26] in view of the arguments they adopt to explain the differences between the $(g-2)$ theoretical and experimental results via a nonstandard geometry. They propose that such a deviation could also be explained by changes of the dimension of the measure and thus the Hausdorff dimension of space-time, which is closely related to our assumptions here.

Here, we claim that the use of an approach of FC based on a sequential form of the modified Riemann-Liouville (MRL) fractional calculus [27] is more appropriate to describe the dynamics associated with field theory and particle physics in the space of nondifferentiable solution functions, or in a coarse-grained scenario for space-time.

It seems that a reasonable way to probe the classical framework of physics is to highlight that, in our real world, the generic point is not infinitely small (or thin); it rather has a thickness. In a coarse-grained space, a point is not infinitely thin, and, here, this feature is modeled by means of a space in which the generic differential is not $d x$, but rather $(d x)^{\alpha}$, likewise for the time variable $t$. It is noteworthy to emphasize that the notion of fractal space-time was introduced in the $70 \mathrm{~s}$ with the seminal work by Stillinger [28], where the axiomatic basis for spaces with noninteger dimension was set up. Later, the concepts associated to a possible noninteger dimension were reinforced with the work by Zeilinger and Svozil [29], where they take into account the intrinsically unavoidable finite resolution of any physical experiment; also the works [30-32] should be quoted here. Along this line, we recommend the work by Nottale [33], where the notion of a fractal space-time is reassessed. Noninteger differentiability and randomness [34] are mutually related in their nature in such a way that studies on fractals on the one hand and fractional Brownian motion on the other hand are often parallel in the work [33]. A function which is continuous everywhere, but nowhere integer-differentiable, necessarily exhibits random-like or pseudorandom features, in that various samplings of these functions, on the same given interval, will be different. This may explain the huge amount of literature extending the theory of stochastic differential equations to describe stochastic dynamics driven by fractional Brownian motion $[27,35,36]$. In connection with the anomalous properties of space-time with multifractal structure, we recommend the reader to consult the interesting work by Calcagni et al. [37] and references therein. Also, we draw special attention to the efforts in building up a solid foundation for the construction of a suitable geometry and a consistent field theory in fractional spaces [25], multifractals [38], and multiscale [39] space-times.

The great majority of actual classical systems are nonconservative but, in spite of that, the most advanced formalisms of classical mechanics deal only with conservative systems [40, 41]. Dissipation [42], for example, is present even at the microscopic level. There is dissipation in every nonequilibrium or fluctuating process, including dissipative tunneling [43-45] and electromagnetic cavity radiation [46, 47], for instance. In [42], we adopt that a way to suitably treat nonconservative systems is through FC, since it can be shown that, for example, a friction force has its form stemming from a Lagrangian that contains a term proportional to the fractional derivative, which may be a derivative of any noninteger order $[40,41,48,49]$. 
Field theory aspects of nonlinear dynamics are today an important subject of study in different subareas of physics and mathematics, but the real success and radically new understanding of nonlinear processes have acquired body over the past 40 years. This understanding has been inspired by the discovery and insights of chaotic dynamics, where the randomness of physical processes is considered and more precisely when particle trajectories are indistinguishable for random process [50].

In [51] we have argued that the modeling of $\mathrm{TeV}$-physics may demand an approach based on fractal operators and FC, and we claim that, in the realm of complexity, nonlocal theories and memory effects are connected to complexity. We also adopt that FC and the nondifferentiable nature of microscopic dynamics may be connected with time scales.

Here, to achieve our goals, we carefully build up a fractional Dirac equation from underlying fractional Weyl equations written down in terms of the helicity operator. We begin by discussing the fractional angular momentum algebra in a coarse-grained scenario in order to understand the fractional spin operator used to set up the helicity operator. Also, a gauge-covariant fractional derivative is proposed so that, from the gauge transformation of charged matter fields, the gauge transformation of the fractional vector potential can be read off. Then, minimal coupling to the electromagnetic field is naturally achieved by writing down fractional Lagrangian densities in terms of fractional gaugecovariant derivatives of the matter fields. It is important to stress that we are not proposing the matter transformation from the very start [22]; we rather get it as an output, from the matter-gauge coupling together with the gauge field transformation. After all, gauge symmetry is actually intrinsic to vector bosons from which we need to decouple spurious degrees of freedom. Matter fields should feel gauge symmetry indirectly, through their coupling to the gauge fields. This is the line of thought we advocate here. Finally, by working out the nonrelativistic approximation of the fractional Dirac's equation, the fractional version of the Pauli's equation is read off and this yields an explicit expression for the fractional $g$-ratio if an external magnetic field is switched on. We point out that fractional Dirac's equation has been the object of interst of several authors over the past decade [5254].

Our paper is outlined as follows. In Section 2, we consider the mathematical backgrounds, with some expressions of the fractional coarse-grained calculus and the modified Riemann-Liouville fractional derivative. Section 3 contains the fractional angular momentum algebra in a coarse-grained scenario. We should clarify that our Sections 2 and 3 contain no original proposal. They are introduced to settle down definitions and conventions and to prepare results that will be used in the subsequent sections. Section 4 is devoted to the fermionic fractional field equations: Weyl and Dirac fermions are studied and the results for the $g$-ratios of the charged leptons are discussed. In Section 5, we focus on the Gordon Decomposition associated to the Fractional Dirac's equation aiming at identifying the fractional spin current. Finally, in Section 6, we cast our discussions and conclusions.

\section{Mathematical Backgrounds}

In the sequel, we adopt an alternative approach by considering a fractional coarse-grained space-time instead of fractional space functions, meaning that neither the space nor the time is infinitely thin but has instead some "thickness". As the use of certain calculation rules is essential to our approach, we briefly comment on this point, before presenting these rules.

The Riemann-Liouville and Caputo approaches for FC are well known and have their rules rigorously proved, as the reader may find in the standard textbooks [55-59]. These well-tested definitions for fractional derivatives, referred to as Riemann-Liouville and Caputo, have been frequently used for several applications. In spite of their efficacy, they have some dangerous pitfalls. For this reason, an interesting definition for fractional derivative $[60,61]$, the so-called modified Riemann-Liouville (MRL) fractional derivative, has been proposed which is less restrictive than other definitions. Its basic expression is as follows:

$$
\begin{aligned}
& D^{\alpha} f(x)=\lim _{x \rightarrow 0} h^{-\alpha} \sum_{k=0}^{\infty}\left(\begin{array}{l}
\alpha \\
k
\end{array}\right)[f(x+(\alpha-k) h)-f((\alpha-k) h)] \\
&=\frac{1}{\Gamma(1-\alpha)} \frac{d}{d x} \int_{0}^{x}(x-t)^{-\alpha}(f(t)-f(0)) d t \\
& 0<\alpha<1 .
\end{aligned}
$$

But, by strictly referring to the context of modified Riemann-Liouville (MRL) formalism, it seems to us worthy to notice that the chain rule, as well as the Leibniz product rule, has had their validity mathematically proven only recently [62]. To be more precise, in the MRL approach the fractional Taylor expansion [62] is the mathematical basis for the validation of the fractional Leibniz product rule and the modified chain rule. We point out that the fractional Leibniz rule used here is a good approximation that comes from the first two terms of the fractional Taylor series development, which holds only for nondifferentiable functions [60-62]. In a recent article [63], the authors show that the Leibniz rule for fractional derivatives in a coarse-grained medium treated as a Hölder-Space yields a modified chain rule. The latter can be safely applied, in agreement with alternative versions of fractional calculus in this class of spaces or even in the local versions of FC.

Following the the MRL definition, we find that the fractional derivative of a constant is zero; and, next, we can use it for both classes of differentiable as nondifferentiable functions. They are cast as follows [61]:

(i) simple rules:

$$
\begin{array}{r}
D^{\alpha} K=0, \quad D x^{\gamma}=\frac{\Gamma(\gamma+1)}{\Gamma(\gamma+1-\alpha)} x^{\gamma-\alpha}, \quad \gamma>0, \\
(u(x) v(x))^{(\alpha)}=u^{(\alpha)}(x) v(x)+u(x) v^{(\alpha)}(x),
\end{array}
$$

where the last rule equation (3) holds only for nondifferentiable functions $u(x)$ and $v(x)$; 
(ii) simple chain rules:

$$
\frac{d^{\alpha}}{d x^{\alpha}} f[u(x)]=\frac{d^{\alpha} f}{d u^{\alpha}}\left(\frac{d u}{d x}\right)^{\alpha} .
$$

Here $f$ is $\alpha$-differentiable and $u$ is differentiable with respect to $x$ and

$$
\frac{d^{\alpha}}{d x^{\alpha}} f[u(x)]=\frac{d f}{d u} \frac{d^{\alpha} u}{d x^{\alpha}},
$$

which holds when $u$ is not differentiable with respect to $x$ and $f(u(x))$ is not differentiable with respect to $x$ (but it is differentiable with respect to $u$ ).

For further details on the MRL formalism, we suggest the readers to follow $[60,61,64]$ which contain all the foundations for the formulation of a fractional differential geometry in a coarse-grained space.

Here, another comment is pertinent and concerns certain definitions called local fractional derivatives, as the ones introduced by Kolwankar and Gangal [65-67], with several works related to this approach, for example, the works [6870] or the approaches with a Hausdorff derivative and also the treatment with the so-called fractal derivative [71, 72]. All of the mentioned approaches seem to be applicable to power-law phenomena. There is also the recently developed $\alpha$-derivative by Kobelev [73]. Our comment now relates to the similarities between the approaches mentioned above on the one hand and the fractional MRL formalism on the other hand. For nondifferentiable functions, similar rules follow. Also, a definition of local fractional derivative by means of a mathematical limit operation is comparable to the differential form of MRL approach and exhibits similar rules. Then, it seems to us that the MRL formalism is an integral version of the fractal derivatives just mentioned and all of them deserve to be more deeply investigated, under a mathematical and a physical point of view $[72,74,75]$. A better understanding of the exact differences and similarities between the traditional fractional calculi based on Riemann-Liouville or Caputo definitions and those with local fractional calculus or even with fractional $q$-calculus $[15,74,76]$ is necessary to also determine the scope of applicability of each one.

Now that we have set up these fundamental expressions, we are ready to carry out the calculations of our main concern.

\section{Fractional Angular Momentum Algebra}

Here, we will derive the commutation algebra for spin-1/2 particles in a coarse-grained medium.

Since in the MRL approach the chain and Leibniz rules hold, it is not difficult to obtain the commutation relation between position and momentum operators and to define the fractional angular momentum components $[22,23]$; we can write

$$
\widehat{L}_{z}^{\alpha}:=\left[\widehat{x}^{\alpha}, \widehat{p}_{y}^{\alpha}\right], \quad \widehat{L}_{y}^{\alpha}:=\left[\widehat{z}^{\alpha}, \widehat{p}_{x}^{\alpha}\right], \quad \widehat{L}_{x}^{\alpha}:=\left[\widehat{y}^{\alpha}, \widehat{p}_{z}^{\alpha}\right],
$$

where $\widehat{x}^{\alpha}, \widehat{y}^{\alpha}$, and $\widehat{z}^{\alpha}$ stand for the position operators, whereas $\widehat{p}_{x}^{\alpha}, \widehat{p}_{y}^{\alpha}$, and $\widehat{p}_{z}^{\alpha}$ refer to the corresponding momentum operators. An important warning is worthy at this stage: we should confuse the $\alpha$-parameter that is simply a label, as in the set of equations above, with the case where it is a true fractional power, as when it appears in some constants like in the factor $\hbar^{\alpha}$.

Again, one can obtain the commutation relations using the definitions given above and the chain (4), (5), and Leibniz (3) rules for MRL as

$$
\begin{aligned}
& {\left[\widehat{L}_{x}^{\alpha}, \widehat{L}_{y}^{\alpha}\right]=i \Gamma(\alpha+1) \hbar^{\alpha} M_{\alpha} \widehat{L}_{z}^{\alpha},} \\
& {\left[\widehat{L}_{z}^{\alpha}, \widehat{L}_{x}^{\alpha}\right]=i \Gamma(\alpha+1) \hbar^{\alpha} M_{\alpha} \widehat{L}_{y}^{\alpha},} \\
& {\left[\widehat{L}_{y}^{\alpha}, \widehat{L}_{z}^{\alpha}\right]=i \Gamma(\alpha+1) \hbar^{\alpha} M_{\alpha} \widehat{L}_{x}^{\alpha},}
\end{aligned}
$$

where $\sigma_{\mu}=\left(\sigma_{0}, \sigma_{1}, \sigma_{2}, \sigma_{3}\right)=\left(I_{2}, \sigma_{x}, \sigma_{y}, \sigma_{z}\right)$ and the factor $M_{\alpha}$ is not an operator; it is simply a multiplicative factor introduced for the sake of ensuring the right dimensionality of these commutators.

Let us now draw the reader's attention to the different character of the $\alpha$-parameter. In $\hbar^{\alpha}, \alpha$ is a true power. In the factor $M_{\alpha}, \alpha$, it is simply a label. In the position, linear momentum and angular momentum operators, $\alpha$ stands for a label that denotes the fractionality. To build up the algebra, we define a set of operators, as in the sequel.

The square of fractional angular moment operator is defined as in [23]: $\left(\widehat{L}^{\alpha}\right)^{2}=\widehat{L}^{\alpha} \widehat{L}^{\alpha}=\left(\widehat{L}_{x}^{\alpha}\right)^{2}+\left(\widehat{L}_{y}^{\alpha}\right)^{2}+\left(\widehat{L}_{z}^{\alpha}\right)^{2}$. We also define the fractional angular momentum operator $\vec{L}^{\alpha}$ and the fractional raising and lowering operators, $L_{+}^{\alpha}$, and their hermitian conjugates $L_{-}^{\alpha}$, respectively, as

$$
\begin{gathered}
\vec{L}^{\alpha} \equiv \widehat{L}_{x}^{\alpha} \widehat{i}+\widehat{L}_{y}^{\alpha} \widehat{j}+\widehat{L}_{z}^{\alpha} \widehat{k}, \\
\widehat{L}_{+}^{\alpha} \equiv \widehat{L}_{x}^{\alpha}+i \widehat{L}_{y}^{\alpha}, \quad L_{-}^{\alpha} \equiv \widehat{L}_{x}^{\alpha}-i \widehat{L}_{y}^{\alpha} .
\end{gathered}
$$

The commutation relations then follow as below:

$$
\begin{gathered}
{\left[\widehat{L}_{+}^{\alpha}, \widehat{L}_{-}^{\alpha}\right]=2 \Gamma(\alpha+1) \hbar^{\alpha} M_{\alpha} \widehat{L}_{z}^{\alpha},} \\
{\left[\widehat{L}_{z}^{\alpha}, \widehat{L}_{+}^{\alpha}\right]=\Gamma(\alpha+1) \hbar^{\alpha} M_{\alpha} \widehat{L}_{+}^{\alpha},} \\
{\left[\widehat{L}_{z}^{\alpha}, \widehat{L}_{-}^{\alpha}\right]=-\Gamma(\alpha+1) \hbar^{\alpha} M_{\alpha} \widehat{L}_{-}^{\alpha},} \\
{\left[\widehat{L}^{\alpha} \widehat{L}^{\alpha}, \widehat{L}_{+}^{\alpha}\right]=\left[\widehat{L}^{\alpha} \widehat{L}^{\alpha}, \widehat{L}_{-}^{\alpha}\right]=\left[\widehat{L}^{\alpha} \widehat{L}^{\alpha}, \widehat{L}_{x}^{\alpha}\right]} \\
=\left[\widehat{L}^{\alpha} \widehat{L}^{\alpha}, \widehat{L}_{y}^{\alpha}\right]=\left[\widehat{L}^{\alpha} \widehat{L}^{\alpha}, \widehat{L}_{z}^{\alpha}\right]=0 .
\end{gathered}
$$

The commutation relations written above indicate that the ordinary integer angular momentum algebra does not change. This implies that the raising (lowering) operator, acting on an eigenstate $|j, m\rangle$ of the $\widehat{L}_{z}^{\alpha}$-operator, raises (lowers) the eigenvalue of the latter by the amount $\Gamma(\alpha+$ 1) $\hbar^{\alpha} M_{\alpha}$. That is, upon the action of the raising (lowering) operator, the eigenvalue of $\widehat{L}_{z}^{\alpha}$ is raised (lowered) by $\Gamma(\alpha+$ 1) $\hbar^{\alpha} M_{\alpha}$.

Another important conclusion is that, for the ordinary Pauli spin matrices, the representation for the basis of $\left(\widehat{L}^{\alpha}\right)^{2}$ is the same as the integer case. This allows us to rewrite all usual 
relations from ordinary quantum mechanics spin algebra in a coarse-grained scenario. The only difference is that, instead of $\hbar$, we have to replace it in all relations by an effective factor $\hbar_{\text {eff }}=\Gamma(\alpha+1) \hbar^{\alpha} M_{\alpha}$. There is no fractional number of particles but there exists an effective Planck constant.

\section{Fractional Field Equations: Weyl and Dirac Fermions}

This section sets out to show our proposal for building fractional versions of Weyl and Dirac equations on the basis of fundamental principles. Rather than adopting a fractionalized version for these equations from the very beginning, we propose to extend the concept of helicity to account for fractionality and, once the suitable helicity operators are written down, we adopt them to propose the fractional Weyl equation for left- and the right-handed fermions. Next, by coupling the different fractional Weyl equations by means of a mass parameter, we will end up with what we propose to be the fractional Dirac equation.

Weyl's equation is a relativistic wave equation to describe massless spin- $1 / 2$ particles. Recalling that helicity is the projection of the spin onto the direction of momentum, we proceed to write down Weyl's equations as statements on the helicity of the left- and right-handed fermions, since, in the case of massless fermions, chirality and helicity are equivalent.

The projection of spin onto the linear momentum is written as follows:

$$
\lambda_{\alpha}=\overrightarrow{S^{\alpha}} \circ \frac{\overrightarrow{p^{\alpha}}}{\left|\overrightarrow{p^{\alpha}}\right|}, \quad \lambda_{\alpha}= \pm 1,
$$

with the spin vector, $\overrightarrow{S_{\alpha}}$, obtained from the angular momentum algebra, which reads

$$
\overrightarrow{S^{\alpha}}=\frac{\hbar^{\alpha}}{2} \Gamma(\alpha+1) M_{\alpha} \vec{\sigma} .
$$

Here the Pauli matrices are the same since the structure of the algebra is not modified, as shown in Section 3.

For a massless particle, the relativistic relation between energy and momentum reads as given below [77]:

$$
E^{\alpha}=\left|\vec{p}^{\alpha}\right| c^{\alpha}
$$

where, in the $c^{\alpha}$ factor, the $\alpha$-parameter plays the role of a power; it is not just a superscript. As before, the $\alpha$ appearing in $\overrightarrow{p^{\alpha}}$ and in the energy operator $E^{\alpha}$ corresponds to a label.

Let us consider a $2 \times 1$ component fermionic field $\chi_{L, \alpha}$ that transforms under a spinor representation of the spacetime group. Taking into account the equations above, we can write

$$
\begin{aligned}
& \left(\hbar^{\alpha} / 2\right) \Gamma(\alpha+1) M_{\alpha} \vec{\sigma} \circ \vec{p}^{\alpha} \chi_{L, \alpha}=\left(\hbar^{\alpha} / 2\right) \Gamma(\alpha+1) M_{\alpha} \chi_{L, \alpha} \\
& \text { or } \quad c^{\alpha} \vec{\sigma} \circ \vec{p}^{\alpha} \chi_{L}=\left|\vec{p}^{\alpha}\right| \chi_{L, \alpha}=E^{\alpha} \chi_{L, \alpha} .
\end{aligned}
$$

To get a quantum wave equation, we invoke the correspondence principle and we propose the fractional operators that represent energy and momentum to be given by the equations that follow:

$$
\begin{gathered}
\widehat{E}^{\beta}=i(\hbar)^{\beta} \frac{\partial^{\beta}}{\partial t^{\beta}}, \\
\widehat{p}^{\alpha}=-i(\hbar)^{\alpha} M_{\alpha} \frac{\partial^{\alpha}}{\partial x^{\alpha}},
\end{gathered}
$$

where the constant $M_{\alpha}$ is included for dimensional reasons, as before. We remark here that if, in the definition of the fractional moment operator (15), the complex $i$ is replaced by an $i^{\alpha}$, the canonical commutation relations change.

By virtue of the correspondence principle, (13) can be rewritten as

$$
i(\hbar)^{\alpha} \vec{\sigma} \circ M_{\alpha} \frac{\partial^{\alpha}}{\partial x^{\alpha}} \chi_{L, \alpha}+i(\hbar)^{\alpha} \frac{M_{t, \alpha}}{c^{\alpha}} \frac{\partial^{\alpha}}{\partial t^{\alpha}} \chi_{L, \alpha}=0,
$$

$M_{t, \alpha}$ is included for dimensional reasons, as already stated above. Here, we are considering space and time with the same fractionality.

The Weyl equation above can be cast in a covariant form as it follows below:

$$
i(\hbar)^{\alpha} \sigma^{\mu} \partial_{\mu}^{(\alpha)} \chi_{L, \alpha}=0 .
$$

Here, $\sigma^{\mu}=\left(\sigma^{0}, \sigma^{i}\right)=\left(\sigma^{0}, \sigma^{1}, \sigma^{2}, \sigma^{3}\right)=\left(I_{2}, \sigma_{x}, \sigma_{y}, \sigma_{z}\right)$ are the usual Pauli-spin matrices and we define the spacetime fractional derivative in Minkowsky space as $\partial_{\mu}^{(\alpha)}=$ $\left(\left(M_{t, \alpha} / c^{\alpha}\right)\left(\partial^{\alpha} / \partial t^{\alpha}\right) ; M_{\alpha}\left(\partial^{\alpha} / \partial x^{\alpha}\right)\right)$.

Also, defining the conjugated Pauli-spin matrices as $\bar{\sigma}^{\mu}=$ $\left(\sigma^{0},-\sigma^{i}\right)$, the following properties hold:

$$
\operatorname{tr}\left(\sigma^{\mu} \bar{\sigma}^{\nu}\right)=\operatorname{tr}\left(\bar{\sigma}^{\mu} \sigma^{\nu}\right)=2 \eta^{\mu \nu}
$$

or

$$
\begin{aligned}
& \sigma^{\mu} \bar{\sigma}^{\nu}+\sigma^{\nu} \bar{\sigma}^{\mu}=2 \eta^{\mu \nu} \mathbf{1}, \\
& \bar{\sigma}^{\mu} \sigma^{\nu}+\bar{\sigma}^{\nu} \sigma^{\mu}=2 \eta^{\mu \nu} \mathbf{1} .
\end{aligned}
$$

Inserting $\bar{\sigma}^{v} \partial_{\nu}^{(\alpha)}$ in (17), we obtain

$$
i(\hbar)^{\alpha} \bar{\sigma}^{\nu} \partial_{\nu}^{(\alpha)} \sigma^{\mu} \partial_{\mu}^{(\alpha)} \chi_{L, \alpha}=0 .
$$

Next, we symmetrize in $(\mu, \nu)$ and make use of $(20)$

The last equation can be rewritten, with the help of the properties of $\sigma^{\mu}$ as $i(\hbar)^{\alpha} \eta^{\mu \nu} \partial_{\nu}^{(\alpha)} \partial_{\mu}^{(\alpha)} \chi_{L, \alpha}=0$ which yields the propagating fractional wave equation for left-helicity fermion $i(\hbar)^{\alpha} \square^{(\alpha)} \chi_{L, \alpha}=0$. The notation for the box symbol is not to be confused with the fractional power operator in distribution theory. Here, the box symbol is defined as $\square^{(\alpha)} \equiv$ $\left(M_{t, \alpha} / c^{\alpha}\right)\left(\partial^{\alpha} / \partial t^{\alpha}\right)\left(\partial^{\alpha} / \partial t^{\alpha}\right)-M_{\alpha}^{2}\left(\partial^{\alpha} / \partial x^{\alpha}\right)\left(\partial^{\alpha} / \partial x^{\alpha}\right)$.

Right-Helicity. A similar procedure can be used to build up a projection for right-chirality fermion by taking in (10) $\lambda_{\alpha}=$ -1 .

Now, the spinor field is noted as $\xi_{R}$ and belongs to a group representation such as $\xi_{R} \in(0 ; 1 / 2)$. We are then ready to write down Weyl's equation for the right-handed sector:

$$
i(\hbar)^{\alpha} \bar{\sigma}^{\mu} \partial_{\mu}^{(\alpha)} \xi_{R, \alpha}=0,
$$


which also conducts us to the equation

$$
i(\hbar)^{\alpha} \square^{(\alpha)} \xi_{R, \alpha}=0 \text {. }
$$

In compact notation, the fractional derivative is given by $\partial_{\mu}^{\alpha}=\left(\left(M_{t, \alpha} / c^{\alpha}\right) \partial_{t}^{\alpha} ; M_{\alpha} \nabla^{\alpha}\right)$.

The two Weyl equations can be written in a more compact form in terms of a four-dimensional spinorial field $\Psi_{\alpha}$ as

$$
i \hbar^{\alpha}\left(\begin{array}{cc}
\mathbf{0} & \bar{\sigma}^{\mu} \partial_{\mu}^{\alpha} \\
\sigma^{\mu} \partial_{\mu}^{\alpha} & \mathbf{0}
\end{array}\right)\left(\begin{array}{l}
\chi_{L, \alpha} \\
\xi_{R, \alpha}
\end{array}\right)=i \hbar^{\alpha} \gamma^{\mu} \partial_{\mu}^{\alpha} \Psi_{\alpha}=0
$$

where $\gamma^{\mu}$ are the usual Dirac gamma-matrices.

We go ahead now by introducing a mass parameter that mixes up the two chiral components to obtain an equation that describes the dynamics of a charged massive fermion. To achieve that, we present the two fractional Weyl equations as

$$
\begin{aligned}
& i(\hbar)^{\alpha} \sigma^{\mu} \partial_{\mu}^{(\alpha)} \chi_{L, \alpha}+u^{\alpha} c^{\alpha} \xi_{R, \alpha}=0, \\
& i(\hbar)^{\alpha} \bar{\sigma}^{\mu} \partial_{\mu}^{(\alpha)} \xi_{R, \alpha}+\widetilde{u}^{\alpha} c^{\alpha} \chi_{L, \alpha}=0,
\end{aligned}
$$

where $u^{\alpha}$ and $\tilde{u}^{\alpha}$ are parameters which, as we will show below, will combine to give the mass of the charged fermion. Imposing that the equations above be compatible with the fractional energy-momentum relationship [77], we are led to

$$
E^{2 \alpha}=p^{2 \alpha} c^{2 \alpha}+m^{2 \alpha} c^{4 \alpha} .
$$

From the second of (25), we obtain

$$
\chi_{L, \alpha}=-\frac{i \hbar^{\alpha}}{\widetilde{u}^{\alpha} c^{\alpha}} \bar{\sigma}^{\mu} \partial_{\mu}^{(\alpha)} \xi_{R, \alpha}
$$

which, once replaced in the first of those equations, yields

$$
\hbar^{2 \alpha} \sigma^{\mu} \partial_{\mu}^{(\alpha)} \bar{\sigma}^{\nu} \partial_{\nu}^{(\alpha)} \xi_{R, \alpha}+u^{\alpha} \widetilde{u}^{\alpha} c^{2 \alpha} \xi_{R, \alpha}=0
$$

or

$$
\square^{(\alpha)} \xi_{R, \alpha}+\frac{u^{\alpha} \widetilde{u}^{\alpha} c^{2 \alpha}}{\hbar^{2 \alpha}} \xi_{R, \alpha}=0 .
$$

This equation indicates, upon comparison with a fractional Klein-Gordon [77] equation, that $u^{\alpha}=\widetilde{u}^{\alpha}=-m^{\alpha}$. With these observations, the fractional Dirac equation can finally be written, from (25), in the general case $\alpha \neq 1$ as

$$
\left(i \hbar^{\alpha} \gamma^{\mu} \partial_{\mu}^{\alpha}-1 m^{\alpha} c^{\alpha}\right) \Psi_{\alpha}=0 .
$$

4.1. Field Transformation, Minimal Coupling, and the GaugeCovariant Fractional Derivative. We start off with the conjugated Dirac's equation:

$$
\bar{\Psi}_{\alpha}\left(-i \hbar^{\alpha} \gamma^{\mu} \partial_{\mu}^{\alpha}-1 m^{\alpha} c^{\alpha}\right)=0
$$

Now, multiplying (30) by $\bar{\Psi}$ and (31) by $\Psi$ and then subtracting them, we obtain

$$
\partial_{\mu}^{\alpha} \bar{\Psi}_{\alpha} \Psi_{\alpha} \gamma^{\mu}+\bar{\Psi}_{\alpha} \gamma^{\mu} \partial_{\mu}^{\alpha} \Psi_{\alpha}=\partial_{\mu}^{\alpha}\left(\bar{\Psi}_{\alpha} \gamma^{\mu} \Psi_{\alpha}\right)=0
$$

The quantity in the bracket can be identified as a conserved current and this equation is interpreted as a fractional continuity equation. From standard electromagnetism coupling with matter, we now take that $j_{\mu} j^{\mu}$, the electromagnetic current, is coupled to the electromagnetic field, $A^{\mu}$, where $j_{\mu}$ is of the form $j_{\mu}=e_{\alpha} \bar{\Psi} \gamma^{\mu} \Psi, e_{\alpha}$ is the electron charge measured in the coarse-grained medium, and $\alpha$ is here simply a label.

For the fractional case, we can think of a minimal coupling term as $e_{\alpha} \bar{\Psi}_{\alpha} \gamma^{\mu} \Psi_{\alpha} A_{\mu}^{\alpha}$, where $A_{\mu}^{\alpha}=\left(\phi^{\alpha} ;-\overrightarrow{A^{\alpha}}\right)$ is the fractional potential tensor transform under Gauge transform as $\left(A_{\mu}^{\alpha}\right)^{\prime}=A_{\mu}^{\alpha}+\partial_{\mu}^{\alpha} \chi$.

Following the Lagrangian for the model with integer dimensions, we can write down the Lagrangian with electromagnetic coupling in the fractional case as given by

$$
\mathscr{L}=\bar{\Psi}_{\alpha}\left(i \hbar^{\alpha} \gamma^{\mu} \partial_{\mu}^{\alpha}-1 m^{\alpha} c^{\alpha}\right) \Psi_{\alpha}-e_{\alpha} \bar{\Psi}_{\alpha} \gamma^{\mu} \Psi_{\alpha} A_{\mu}^{\alpha}
$$

which can be re written as

$$
\mathscr{L}=\bar{\Psi}_{\alpha} i \hbar^{\alpha} \gamma^{\mu}\left(\partial_{\mu}^{\alpha}+\frac{i e_{\alpha}}{\hbar^{\alpha}} A_{\mu}^{\alpha}\right) \Psi_{\alpha}-1 m^{\alpha} c^{\alpha} \bar{\Psi}_{\alpha} \Psi_{\alpha} .
$$

In order that the theory remains covariant, we take the spinor field transformation as follows:

$$
\Psi_{\alpha}^{\prime}=R(\chi) \Psi_{\alpha}
$$

where $R(\chi)$ is taken to be unitary, but its explicit form will be determined in the sequel. The Lagrangian density with the electromagnetic coupling term suggests the definition of the fractional gauge-covariant derivative to be of the form

$$
D_{\mu}^{\alpha}=\partial_{\mu}^{\alpha}+\frac{i e_{\alpha}}{c^{\alpha} \hbar^{\alpha}} k A_{\mu}^{\alpha}
$$

where the tensor field $A_{\mu}^{\alpha}$ is considered to have a fractional Gage symmetry and transforms by

$$
\left(A_{\mu}^{\alpha}\right)^{\prime}=A_{\mu}^{\alpha}+\partial_{\mu}^{\alpha} \chi
$$

The fractional covariant derivative of the field $\Psi_{\alpha}$ obeys, as usually done in the approach of gauge theories, the same transform rule as the matter field:

$$
\left(D_{\mu}^{\alpha} \Psi_{\alpha}\right)^{\prime}=R(\chi) D^{\alpha} \Psi_{\alpha}
$$

In more details,

$$
\partial_{\mu}^{\alpha} \Psi_{\alpha}^{\prime}+\frac{i e_{\alpha}}{c^{\alpha} \hbar^{\alpha}} k A_{\mu}^{\alpha \prime} \Psi_{\alpha}^{\prime}=R(\chi) \partial_{\mu}^{\alpha} \Psi_{\alpha}+\frac{i e_{\alpha}}{c^{\alpha} \hbar^{\alpha}} k A_{\mu}^{\alpha} \Psi_{\alpha}
$$

which turn into a fractional differential equation of the form

$$
\frac{\partial_{\mu}^{\alpha} R}{R}=-\frac{i e_{\alpha}}{c^{\alpha} \hbar^{\alpha}} \partial_{\mu}^{\alpha} \chi
$$

The solution to the equation above reads

$$
R=\exp \left(-\frac{i e_{\alpha}}{c^{\alpha} \hbar^{\alpha}} \chi\right)
$$


This can be readily proven by means of the following the steps: fractionally deriving the above equation, with the use of (5), we get that

$$
\partial_{\mu}^{\alpha} R=-\frac{i e_{\alpha}}{c^{\alpha} \hbar^{\alpha}} \exp \left(-\frac{i e_{\alpha}}{c^{\alpha} \hbar^{\alpha}} \chi\right) \partial_{\mu}^{\alpha} \chi=-\frac{i e_{\alpha}}{c^{\alpha} \hbar^{\alpha}} R \partial_{\mu}^{\alpha} \chi
$$

and this proves our assertion.

The fractional Dirac equation, in a coarse-grained scenario, may now be written with the minimal electromagnetic coupling as

$$
i \hbar^{\alpha} \gamma^{\mu}\left(\partial_{\mu}^{\alpha}+\frac{i e_{\alpha}}{c^{\alpha} \hbar^{\alpha}} A_{\mu}^{\alpha}\right) \Psi_{\alpha}-1 m^{\alpha} c^{\alpha} \Psi_{\alpha}=0
$$

Splitting the space and time components, multiplying by $\gamma^{0}$ and using the properties of the Dirac gamma-matrices, we obtain

$$
\begin{gathered}
i \hbar^{\alpha} \gamma^{0} \gamma^{0} \frac{1}{c^{\alpha}} \partial_{t}^{\alpha} \Psi_{\alpha}+i \hbar^{\alpha} \gamma^{0} \gamma^{i} M_{x, \alpha} \partial_{x}^{\alpha} \Psi_{\alpha}+i \hbar^{\alpha} \gamma^{0} \gamma^{0} \frac{i e_{\alpha}}{c^{\alpha} \hbar^{\alpha}} \phi^{\alpha} \Psi_{\alpha} \\
+i \hbar^{\alpha} \gamma^{0} \gamma^{i} \frac{i e_{\alpha}}{c^{\alpha} \hbar^{\alpha}}\left(-\vec{A}^{\alpha}\right) \Psi_{\alpha}-\gamma^{0} m^{\alpha} c^{\alpha} \Psi_{\alpha}=0
\end{gathered}
$$

or

$$
\begin{aligned}
i \hbar^{\alpha} 1 \partial_{t}^{\alpha} \Psi_{\alpha}= & c^{\alpha} \gamma^{0} \gamma^{i}\left(-i \hbar^{\alpha} M_{x, \alpha} \partial_{x}^{\alpha} \Psi_{\alpha}-\frac{e_{\alpha}}{c^{\alpha}} \overrightarrow{A^{\alpha}} \Psi_{\alpha}\right) \\
& +\gamma^{0} \gamma^{0} e_{\alpha} \phi^{\alpha} \Psi_{\alpha}+\gamma^{0} m^{\alpha} c^{2 \alpha} \Psi_{\alpha}, \\
i \hbar^{\alpha} 1 \partial_{t}^{\alpha} \Psi_{\alpha}= & c^{\alpha} \gamma^{0} \gamma^{i}\left(-i \hbar^{\alpha} M_{x, \alpha} \partial_{x}^{\alpha} \Psi_{\alpha}-\frac{e_{\alpha}}{c^{\alpha}} \overrightarrow{A^{\alpha}} \Psi_{\alpha}\right) \\
& +1 e_{\alpha} \phi^{\alpha} \Psi_{\alpha}+\gamma^{0} m^{\alpha} c^{2 \alpha} \Psi_{\alpha} .
\end{aligned}
$$

Using the correspondence principle, we have

$$
\begin{aligned}
i \hbar^{\alpha} 1 \partial_{t}^{\alpha} \Psi_{\alpha}= & c^{\alpha} \vec{\alpha} \circ\left(\vec{p}^{\alpha}-\frac{e_{\alpha}}{c^{\alpha}} \overrightarrow{A^{\alpha}}\right) \Psi_{\alpha} \\
& +\hbar^{\alpha} 1 e_{\alpha} \phi^{\alpha} \Psi_{\alpha}+\gamma^{0} m^{\alpha} c^{2 \alpha} \Psi_{\alpha} .
\end{aligned}
$$

Now, we write the Dirac spinor as a column-vector in the coarse-grained scenario as

$$
\Psi_{\alpha}=\left(\begin{array}{c}
\psi_{\alpha, s} \\
\psi_{\alpha, w}
\end{array}\right)
$$

where we have named the strong $(s)$ and weak $(w)$ components as the bispinors: $\psi_{\alpha, s}=\left(\begin{array}{l}\psi_{\alpha, 1} \\ \psi_{\alpha, 2}\end{array}\right), \psi_{\alpha, w}=\left(\begin{array}{l}\psi_{\alpha, 3} \\ \psi_{\alpha, 4}\end{array}\right)$, respectively. In a symplectic form, we can write

$$
\begin{gathered}
\psi_{\alpha, s}=\psi_{\alpha, 1}+i \psi_{\alpha, 2} \\
\psi_{\alpha, w}=\psi_{\alpha, 3}-i \psi_{\alpha, 4}
\end{gathered}
$$

4.2. The Nonrelativistic Regime of Fractional Dirac's Equation and the Fractional g-Ratio. In order to work out the nonrelativistic limit of the fractional Dirac's Equation, we have to consider that the dominant term in the Hamiltonian is the rest energy, given by $m^{\alpha} c^{2 \alpha}$. We then propose an ansatz for the solution to the fractional Dirac's equation as

$$
\Psi_{\alpha}^{\prime}=e^{\left(-i\left(E^{\alpha} / \hbar^{\alpha}\right) t^{\alpha}\right)}\left(\begin{array}{c}
\psi_{\alpha, s} \\
\psi_{\alpha, w}
\end{array}\right), \quad E^{\alpha} \cong m^{\alpha} c^{2 \alpha} .
$$

Inserting this ansatz into the fractional equation (30), with the help of the chain rule (4) and the Leibniz rule (3), we obtain

$$
\begin{aligned}
i \hbar^{\alpha} 1 \partial_{t}^{\alpha}\left(\begin{array}{c}
\psi_{\alpha, s} \\
\psi_{\alpha, w}
\end{array}\right)= & \left(\begin{array}{c}
c^{\alpha} \vec{\sigma} \circ\left(\vec{p}^{\alpha}-\frac{e_{\alpha}}{c^{\alpha}} \vec{A}^{\alpha}\right) \\
c^{\alpha} \vec{\sigma} \circ\left(\overrightarrow{p^{\alpha}}-\frac{e_{\alpha}}{c^{\alpha}} \vec{A}^{\alpha}\right)
\end{array}\right)\left(\begin{array}{c}
\psi_{\alpha, w} \\
\psi_{\alpha, s}
\end{array}\right) \\
& +e_{\alpha} \phi^{\alpha}\left(\begin{array}{c}
\psi_{\alpha, s} \\
\psi_{\alpha, w}
\end{array}\right)+m^{\alpha} c^{2 \alpha}\left(\begin{array}{c}
\psi_{\alpha, s} \\
-\psi_{\alpha, w}
\end{array}\right) \\
& -m^{\alpha} c^{2 \alpha} \Gamma(\alpha+1)\left(\begin{array}{c}
\psi_{\alpha, s} \\
\psi_{\alpha, w}
\end{array}\right),
\end{aligned}
$$

which yields two equations as cast below:

$$
\begin{aligned}
i \hbar^{\alpha} 1 \partial_{t}^{\alpha} \psi_{\alpha, b}= & c^{\alpha} \vec{\sigma} \circ\left(\overrightarrow{p^{\alpha}}-\frac{e_{\alpha}}{c^{\alpha}} \overrightarrow{A^{\alpha}}\right) \psi_{\alpha, w}+e_{\alpha} \phi^{\alpha} \psi_{\alpha, s} \\
& +(1-\Gamma(\alpha+1)) m^{\alpha} c^{2 \alpha} \psi_{\alpha, s}, \\
i \hbar^{\alpha} 1 \partial_{t}^{\alpha} \psi_{\alpha, w}= & c^{\alpha} \vec{\sigma} \circ\left(\overrightarrow{p^{\alpha}}-\frac{e_{\alpha}}{c^{\alpha}} \vec{A}^{\alpha}\right) \psi_{\alpha, s}+e_{\alpha} \phi^{\alpha} \psi_{\alpha, w} \\
& -(1+\Gamma(\alpha+1)) m^{\alpha} c^{2 \alpha} \psi_{\alpha, w} .
\end{aligned}
$$

We now consider that the mass term is dominant over the electrostatic interaction energy, that is, $e_{\alpha} \phi^{\alpha} \lll m^{\alpha} c^{2 \alpha}(1+$ $\Gamma(\alpha+1))$, and that the weakly relativistic component $\psi_{\alpha, w}$ has slow evolution whenever compared to the rest energy, $i \hbar^{\alpha} 1 \partial_{t}^{\alpha} \psi_{\alpha, w}<m^{\alpha} c^{2 \alpha}(1+\Gamma(\alpha+1)) \psi_{\alpha, w}$. With these approximations, we can write that the second equation takes the form

$$
\begin{aligned}
0 \cong & c^{\alpha} \vec{\sigma} \circ\left(\vec{p}^{\alpha}-\frac{e_{\alpha}}{c^{\alpha}} \vec{A}^{\alpha}\right) \psi_{\alpha, s}+e_{\alpha} \phi^{\alpha} \psi_{\alpha, w} \\
& -(1+\Gamma(\alpha+1)) m^{\alpha} c^{2 \alpha} \psi_{\alpha, w},
\end{aligned}
$$

which leads to the relation between the relativistic weak and strong components as

$$
\psi_{\alpha, w} \cong \frac{c^{\alpha} \vec{\sigma} \circ\left(\vec{p}^{\alpha}-\left(e_{\alpha} / c^{\alpha}\right) \vec{A}^{\alpha}\right)}{(1+\Gamma(\alpha+1)) m^{\alpha} c^{2 \alpha}} \psi_{\alpha, s} .
$$

The expression above readily gives that the weak component is, within the approximations carried out above, suppressed with respect to the strong bispinor, $\psi_{\alpha, w} \lll \psi_{\alpha, s}$.

Now, inserting this result into the first equation (51), the equation for the strong component turns out to be

$$
\begin{aligned}
i \hbar^{\alpha} 1 \partial_{t}^{\alpha} \psi_{\alpha, s} & \\
= & \frac{\vec{\sigma} \circ\left(\vec{p}^{\alpha}-\left(e_{\alpha} / c^{\alpha}\right) \vec{A}^{\alpha}\right) \vec{\sigma} \circ\left(\vec{p}^{\alpha}-\left(e_{\alpha} / c^{\alpha}\right) \vec{A}^{\alpha}\right)}{(1+\Gamma(\alpha+1)) m^{\alpha}} \psi_{\alpha, s} \\
& +e_{\alpha} \phi^{\alpha} \psi_{\alpha, s}+(1-\Gamma(\alpha+1)) m^{\alpha} c^{2 \alpha} \psi_{\alpha, s} .
\end{aligned}
$$


We define the fractional momentum operator as $\vec{\pi}^{\alpha} \equiv$ $\vec{p}^{\alpha}-\left(e_{\alpha} / c^{\alpha}\right) \overrightarrow{A^{\alpha}}$, and we use the well-known propriety of Pauli matrices $\left(\vec{\sigma} \circ \pi^{\alpha}\right)\left(\vec{\sigma} \circ \pi^{\alpha}\right)=\pi^{\alpha} \circ \pi^{\alpha}+i \vec{\sigma} \circ\left(\pi^{\alpha} \wedge \pi^{\alpha}\right)$ and also $\left(\pi^{\alpha} \wedge \pi^{\alpha}\right)=-\left(e_{\alpha} / c^{\alpha}\right) \vec{p}^{\alpha} \wedge \vec{A}^{\alpha}$. Now, with the definition $\vec{p}^{\alpha}=$ $i \hbar^{\alpha} M_{x, \alpha} \vec{\nabla}^{\alpha}, \vec{\nabla}^{\alpha} \equiv \widehat{i}\left(\partial^{\alpha} / \partial x^{\alpha}\right)+\widehat{j}\left(\partial^{\alpha} / \partial y^{\alpha}\right)+\widehat{k}\left(\partial^{\alpha} / \partial z^{\alpha}\right)$ and in analogy with the integer case, $\vec{\nabla}^{\alpha} \wedge{\overrightarrow{A^{\alpha}}}^{\alpha}=\vec{B}^{\alpha}$, we can write down

$$
\begin{aligned}
i \hbar^{\alpha} 1 \partial_{t}^{\alpha} \psi_{\alpha, b}= & \frac{\left(\overrightarrow{p^{\alpha}}-\left(e_{\alpha} / c^{\alpha}\right) \vec{A}^{\alpha}\right)^{2}}{(1+\Gamma(\alpha+1)) m^{\alpha}} \psi_{\alpha, b} \\
& -\frac{\hbar^{\alpha} M_{x, \alpha}\left(e_{\alpha} / c^{\alpha}\right) \vec{\sigma} \circ \vec{B}^{\alpha}}{(1+\Gamma(\alpha+1)) m^{\alpha}} \psi_{\alpha, b}+e_{\alpha} \phi^{\alpha} \psi_{\alpha, b} \\
& +(1-\Gamma(\alpha+1)) m^{\alpha} c^{2 \alpha} \psi_{\alpha, b} .
\end{aligned}
$$

Equation (55) is the fractional version of the Pauli equation in a coarse-grained scenario.

Recalling that the spin term is $\vec{S}_{\alpha}=\left(\hbar^{\alpha} / 2\right) \Gamma(\alpha+1) M_{x, \alpha} \vec{\sigma}$, the second term in the r.h.s. of the above equation can be written as

$$
-\frac{e_{\alpha}}{2 m^{\alpha} c^{\alpha}}\left(\frac{4}{(1+\Gamma(\alpha+1)) \Gamma(\alpha+1)}\right) \vec{S}_{\alpha} \circ \vec{B}^{\alpha} \psi_{\alpha, s} .
$$

The factor into the bracket can be identified as the fractional gyromagnetic ratio or $g$-factor $g_{\text {frac }}$ :

$$
g_{\mathrm{frac}}=\frac{4}{(1+\Gamma(\alpha+1)) \Gamma(\alpha+1)} .
$$

Notice that (57) has a universal form in that it is the same for a general $\alpha$. However, as we will be discussing in Section 6 , $\alpha$ is species-dependent: electron, muon, and the tau will exhibit different fractionality, that is, different $\alpha$-parameters. In this sense, we may say that (57) is species-dependent. This remark will be very important for a relationship that will be proposed in Section 6 between the mean life time of the lepton and the $\alpha$-parameter. Notice that, whenever $\alpha=1$, $g_{\text {frac }}=g=2$, the equation turns into the usual Pauli equation.

We can map a fractional parameter with the CODATA [4] known value of $g_{\text {exp }}$ for electrons and muons and according to [78] for the lepton tau. The mapping can be achieved by numerically solving the equation

$$
g_{\mathrm{frac}}=\frac{4}{(1+\Gamma(\alpha+1)) \Gamma(\alpha+1)}=g_{\mathrm{exp}} .
$$

Since we expect the $\alpha$-parameter to be very close to 1 (low fractionality), we could write that $\alpha=1-\varepsilon, \varepsilon$ being a deviation from 1 as an effect of the fractionality; then, we could write $g_{\text {frac }}=2+\delta(\varepsilon)$, where $\delta$ is a small deviation. Then, by expanding the gamma functions in the denominator of (58) around $\varepsilon=0, \delta(\varepsilon)$ it could be readily computed. However, our option is to avoid the expansion in $\varepsilon$ in order to have a result that includes all orders in $\varepsilon$ so that it can be compared with a better precision with the experimental value of $g$, written here as $g_{\text {exp }}$. In our Discussion and Conclusions section (Section 6), we will elaborate more on this result and we will show how we make use of it to fit the $g$-ratios of the charged leptonic particles.

We believe that, for the sake of completeness, it would be instructive to present the Gordon decomposition for the electronic current in its fractional form. The Gordon decomposition readily manifests the magnetic dipole piece of the relativistic current. So, after we have studied the effect of the fractionality on the $g$-factor, it is worthy to show explicitly that the spin contribution couples to the electron momentum transfer. We will do that in the next section and we choose to work it out in the configuration space, rather than in momentum space, as one usually does.

\section{The Gordon Decomposition and the Fractional Dirac's Equation: A Fractional Spin Current}

From (32), we can define the fractional current density as

$$
j_{\alpha}^{\mu}=e_{\alpha} \bar{\Psi} \gamma^{\mu} \Psi_{\alpha}
$$

where $\alpha$ here does not stand for the spinor index, as it might appear; $\alpha$ refers to fractionality, as in the previous sections.

The above equation may be rewritten as

$$
j_{\alpha}^{\mu}=\frac{1}{2} e_{\alpha}\left(\bar{\Psi}_{\alpha} \gamma^{\mu} \Psi_{\alpha}+\bar{\Psi}_{\alpha} \gamma^{\mu} \Psi_{\alpha}\right)
$$

From the Dirac equation (30) and its Dirac-conjugated version, (31) we may write, respectively,

$$
\begin{aligned}
& \Psi_{\alpha}=\frac{i \hbar^{\alpha}}{m^{\alpha} c^{\alpha}} \gamma^{\mu} \partial_{\mu}^{\alpha} \Psi_{\alpha}, \\
& \bar{\Psi}_{\alpha}=-\frac{i \hbar^{\alpha}}{m^{\alpha} c^{\alpha}} \partial_{\mu}^{\alpha} \bar{\Psi}_{\alpha} \gamma^{\mu} .
\end{aligned}
$$

Now, inserting these results in the first and second terms of (60), respectively, we can write for the fractional current

$$
j_{\alpha}^{\mu}=\frac{i \hbar^{\alpha} e_{\alpha}}{m^{\alpha} c^{\alpha}}\left(\left(\bar{\Psi}_{\alpha} \gamma^{\mu} \gamma^{\nu}\right)\left(\partial_{\gamma}^{\alpha} \Psi_{\alpha}\right)-\left(\partial_{\nu}^{\alpha} \bar{\Psi}_{\alpha}\right)\left(\gamma^{\nu} \gamma^{\mu} \Psi_{\alpha}\right)\right) \text {. }
$$

For the gamma-matrices, we can write that

$$
\begin{aligned}
\gamma^{\mu} \gamma^{\nu} & =\frac{1}{2}\left(\gamma^{\mu} \gamma^{\nu}+\gamma^{\nu} \gamma^{\mu}\right)+\frac{1}{2}\left(\gamma^{\mu} \gamma^{\nu}-\gamma^{\nu} \gamma^{\mu}\right) \\
& =\eta^{\mu \nu} 1+\Sigma^{\mu \nu} \\
\gamma^{\nu} \gamma^{\mu}= & \eta^{\mu \nu} 1+\Sigma^{\nu \mu}=\eta^{\mu \nu} 1-\Sigma^{\mu \nu},
\end{aligned}
$$

where $\Sigma^{\mu \nu}=(1 / 2)\left(\gamma^{\mu} \gamma^{\nu}-\gamma^{\nu} \gamma^{\mu}\right)=(1 / 2)\left[\gamma^{\mu}, \gamma^{\nu}\right]$ and $\eta^{\mu \nu}$ is the metric tensor.

Using the definitions above and inserting them into (62), we obtain for the current a suitable form, where we split the contribution due to the spin:

$$
\begin{aligned}
j_{\alpha}^{\mu}= & \frac{i \hbar^{\alpha} e_{\alpha}}{2 m^{\alpha} c^{\alpha}}\left(\bar{\Psi}_{\alpha}\left(\partial_{\nu}^{\alpha} \Psi_{\alpha}\right)-\left(\partial_{\nu}^{\alpha} \bar{\Psi}_{\alpha}\right) \Psi_{\alpha}\right) \\
& +\frac{i \hbar^{\alpha} e_{\alpha}}{2 m^{\alpha} c^{\alpha}}\left(\bar{\Psi}_{\alpha} \Sigma^{\mu \nu}\left(\partial_{\nu}^{\alpha} \Psi_{\alpha}\right)+\left(\partial_{\nu}^{\alpha} \bar{\Psi}_{\alpha}\right) \Sigma^{\mu \nu} \Psi_{\alpha}\right) .
\end{aligned}
$$


Defining $\sigma^{\mu \nu}=i \Sigma^{\mu \nu}$, the second term in the equation above may be identified with the spin contribution to the fractional current and reads as

$$
j_{\alpha, \text { spin }}^{\mu}=\frac{\hbar^{\alpha} e_{\alpha}}{2 m^{\alpha} c^{\alpha}} \partial_{\nu}^{\alpha}\left(\bar{\Psi}_{\alpha} \sigma^{\mu \nu} \Psi_{\alpha}\right) .
$$

Therefore, we are here giving the Gordon decomposition carried out in configuration space rather than presenting it in Fourier space. We choose to do so because we are not dealing with generalized functions, but with nondifferentiable functions.

\section{Discussions and Conclusions}

With the help of (58), we are able to compare the the fractional $\alpha$-parameter for electrons, muons, and taus. The numerical results show that the fractionality, $\alpha$, exhibits a hierarchy; that is, $\alpha$ (electron) $>\alpha$ (muon) $>\alpha$ (tau). (In what follows $g_{e \text {-exp }}$ is the $g$-factor for electrons given by experimental CODATA reference. Analogously, for $g_{\mu \text {-exp }}$, the $\alpha_{\mu \text {-exp }}$ parameter is the corresponding one for the fermion species.)

For the electron (CODATA), we have that $g_{e \text {-exp }}=$ 2.00231930436146. This yields, in our approach, $\alpha_{e-\exp }=$ 0.9981697906061296726 , indicating a system with low fractionality ( $\alpha$ near 1$)$.

Analogously, for the muon (CODATA), $g_{\mu \text {-exp }}=2.00233184182$, which gives $\alpha_{\mu \text {-exp }}=$ 0.9981598882409105161 and for the tau-lepton (data from QED; see e.g., [78]), $g_{\tau}=2.00235442$, such that our $\alpha_{\tau}=0.998142055249517567$.

Notice the hierarchy for the $g$-factors: $g_{\tau}>g_{\mu \text {-Exp }}>$ $g_{e-\exp }:$ an inverted hierarchy with respect to the values of $\alpha$ parameters.

We can interpret these results as follows: the higher is the $g$-ratio, the farther is the $\alpha$-parameter from $\alpha=1$ ( $g$-factor is a decreasing function with alpha, as it can be seen numerically from (57)). A larger $\alpha$-parameter means obviously fractionality closer to 1 . Moreover, if this parameter deviates significantly from $\alpha=1$, it may indicate that the system is more sparse, or equivalently, roughness is larger. This may suggest that there is a smaller variety of different types of interactions (particles and fields) in the surroundings of the pseudoparticle. In other words, if a particle interacts less or has a lower mean life than another particle, its corresponding $\alpha$-parameter would be a little more distant (downwards) from $\alpha=1$, which indicates a lower complexity of interactions and also that the medium "seen" by the particle is more rugged. We understand that a value of alpha closer to 1 suggests an enhacement in the complexity of the interactions. By this we mean that the particle interacts more significantly with more structures in its neighborhood. An $\alpha$-parameter equal to 1 means one type of total mixing memory (providing an idealized integer model, which is not natural), with the pseudoparticles feeling the interactions of all kinds, so that, in average, these fluctuations are vanishing (a statistical average). We can speculate that the medium seen by this kind of particle is less rugged or closer to the continuum.
Now, in terms of mean life, muon decays in $10^{-6} \mathrm{~s}$ and tau decays in $10^{-15} \mathrm{~s}$, while the electron is stable. The relation between mean life, the $\alpha$-parameter, and the coarse-grained character can be understood as follows: the lower is the mean life; this means that the particle does not have sufficient time to interact with the surrounding environment and with other particles, implying that the $\alpha$-parameter departs from the value 1 . The overall interaction felt by those particles is limited to the closer interacting particles and the neighbor environment. Thinking this way, the medium appears more rugged for this particle. Stable particles, like electrons, can experience the entire environment; therefor it interacts more, justifying a fractional parameter closer to 1 . So, this medium could be considered less rugged for the electrons than for the other leptonic species. The time scale of the interaction expressed by the mean life can give clues on the fractionality.

From QED calculations $[3,4]$, we also have for electron, muon, and tau (in what follows, $g_{e-\mathrm{QED}}$ is the $g$-factor for electrons given by QED; analogously, for $g_{\mu \text {-QED }}$ and $g_{\mu \text {-QED }}$, the $\alpha_{\mu \text {-QED }}$ parameter is the corresponding one for the fermion species):

$$
\begin{aligned}
g_{e-\mathrm{QED}} & =2.00231930436364, \\
\alpha_{e-\mathrm{QED}} & =0.9981697906044078681 ; \\
g_{\mu-\mathrm{QED}} & =2.002331694362, \\
\alpha_{\mu-\mathrm{QED}} & =0.9981600047070900225 .
\end{aligned}
$$

Comparing the parameters above from QED with those from the measured anomalies [4], we can see that $g_{e-\mathrm{QED}}=$ $2.00231930436364>g_{e-\exp }=2.00231930436146$. This leads to $\alpha_{e-\mathrm{QED}}=0.9981697906044078681<\alpha_{e-\exp }=$ 0.9981697906061296726 .

We can see the opposite behavior for muon: $g_{\mu-\mathrm{QED}}=$ $2.002331694362<g_{\mu \text {-exp }}=2.00233184182$, what leads to $\alpha_{\mu \text {-QED }}=0.9981600047070900225>\alpha_{\mu-\exp }=$ 0.9981598882409105161 .

We can interpret these results as follows: for the electron, the results may be indicating that the complexity of the interactions taken into account in the QED calculation may be lower than it would be in the reality seen by the experiment. That is, the electron, as a pseudoparticle, keeps hidding other interactions or even structures that are not well described or are incomplete in the SM description. Thus, this may justify the deviation from the QED calculations as compared to the experimental results. In other words, in the experimental reality for electrons, we may think that the complexity is larger than that considered in the QED calculations based on the SM and the fractionality should indicate that the SM, although very good, may not be providing all information necessary to describe the interactions in a more complete view, either at high energies or in granular or fractal spacetime. Thus, the FC may give evidences that the SM could require corrections (or higher order calculations by QED).

For the muon, an opposite behavior in terms of fractional parameter could be observed and indicates that the QED calculations might be taken into account with more interactions and a consequent complexity than the particle really 
experience, suggesting that the SM may not be complete to describe the whole interaction scenario.

In summary, in the present work, we have built up a fractional Dirac equation in a coarse-grained scenario by taking into account a fractional Weyl equation, a fractional angular momentum algebra, by introducing a mass parameter and imposing that the equations be compatible with the fractional energy-momentum relation. Considering then a fractional gauge-covariant derivative, we could minimally couple the charged fermion to an external electromagnetic field. The step further consisted in working out the nonrelativistic limit of the Dirac's equation to obtain a fractional version of the Pauli's equation. We have then investigated the anomalous magnetic moment for the charged leptons: electrons, muons, and taus. With the fractional approach to a coarse-grained scenario, we were able to get the fractionality associated to each leptonic species.

We have shown that a mapping of the anomalous magnetic $g$-ratio is possible in terms of a fractional parameter that could be used to give some insight about the precision of the QED calculations necessary to better emulate reality.

Defining a fractional current density, we have also performed a fractional Gordon decomposition and identified the spin contribution to the fractional electromagnetic current density.

We also suggested that the understanding of the results comparatively for electrons, muons and taus may be thought in the realm of complexity, mean life, coarse-grainedness, and pseudoparticle concepts.

We have finally shown that the fractionalities, $\alpha$, exhibit a hierarchy $\alpha$ (electron) $>\alpha$ (muon) $>\alpha$ (tau). We established a connection between mean life of the particle, fractionality, and roughness, showing that a small mean life can lead to a fractional parameter more distant from $\alpha=1$ if compared with more stable particles. Consequently, a small mean life means less interaction, less complexity related to interactions and to the effective medium felt by the particle. This means more roughness.

\section{Conflict of Interests}

The authors declare that there is no conflict of interests regarding the publication of this paper.

\section{Acknowledgments}

Cresus F. L. Godinho is acknowledged for the discussions at an early stage of this work. The authors wish to express their gratitude to FAPERJ-Rio de Janeiro and CNPq-Brazil for the partial financial support.

\section{References}

[1] M. Passera, W. J. Marciano, and A. Sirlin, "The muon g-2 discrepancy: errors or new physics?" in Proceedings of the 16th International Conference on Supersymmetry and the Unification of Fundamental Interactions (SUSY '08), vol. 1078 of AIP Conference Proceedings, pp. 378-381, kor, June 2008.
[2] F. Jegerlehner and A. Nyffeler, "The muon g-2," Physics Reports, vol. 477, no. 1-3, pp. 1-110, 2009.

[3] E. de Rafael, "Update of the electron and muon g-factors," Nuclear Physics B-Proceedings Supplements, vol. 234, pp. 193198, 2013.

[4] P. J. Mohr, B. N. Taylor, and D. B. Newell, "CODATA recommended values of the fundamental physical constants: 2010," Reviews of Modern Physics, vol. 84, no. 4, 2012.

[5] J. P. Miller, E. De Rafael, and B. L. Roberts, "Muon (g-2): experiment and theory," Reports on Progress in Physics, vol. 70, no. 5, pp. 795-881, 2007.

[6] S. J. Brodsky, V. A. Franke, J. R. Hiller, G. McCartor, S. A. Paston, and E. V. Prokhvatilov, "A nonperturbative calculation of the electron's magnetic moment," Nuclear Physics B, vol. 703, no. 1-2, pp. 333-362, 2004.

[7] V. E. Tarasov, "The fractional oscillator as an open system," Central European Journal of Physics, vol. 10, no. 2, pp. 382-389, 2012.

[8] R. Metzler and J. Klafter, “The random walk's guide to anomalous diffusion: a fractional dynamics approach," Physics Report, vol. 339, no. 1, pp. 1-77, 2000.

[9] R. Metzler and J. Klafter, "The restaurant at the end of the random walk: recent developments in the description of anomalous transport by fractional dynamics," Journal of Physics A, vol. 37, no. 31, pp. R161-R208, 2004.

[10] R. Metzler, E. Barkai, and J. Klafter, "Anomalous diffusion and relaxation close to thermal equilibrium: a fractional FokkerPlanck equation approach," Physical Review Letters, vol. 82, no. 18 , pp. 3563-3567, 1999.

[11] M. A. E. Herzallah and D. Baleanu, "Fractional-order EulerLagrange equations and formulation of Hamiltonian equations," Nonlinear Dynamics, vol. 58, no. 1-2, pp. 385-391, 2009.

[12] D. Fulger, E. Scalas, and G. Germano, "Monte Carlo simulation of uncoupled continuous-time random walks yielding a stochastic solution of the space-time fractional diffusion equation," Physical Review E, vol. 77, no. 2, Article ID 021122, 2008.

[13] R. Hilfer, "Experimental evidence for fractional time evolution in glass forming materials," Chemical Physics, vol. 284, no. 1-2, pp. 399-408, 2002.

[14] A. Stanislavsky, K. Weron, and J. Trzmiel, "Subordination model of anomalous diffusion leading to the two-power-law relaxation responses," Europhysics Letters, vol. 91, no. 6, Article ID 40003, 2010.

[15] R. Metzler, W. Schick, H.-G. Kilian, and T. F. Nonnenmacher, "Relaxation in filled polymers: a fractional calculus approach," The Journal of Chemical Physics, vol. 103, no. 16, pp. 7180-7186, 1995.

[16] W. G. Glockle and T. F. Nonnenmacher, "Fractional integral operators and Fox functions in the theory of viscoelasticity," Macromolecules, vol. 24, no. 24, pp. 6426-6434, 1991.

[17] R. E. Gutierrez, J. M. Rosário, and J. T. Machado, "Fractional order calculus: basic concepts and engineering applications," Mathematical Problems in Engineering, vol. 2010, Article ID 375858, 19 pages, 2010.

[18] E. M. C. Abreu and C. F. L. Godinho, "Fractional Dirac bracket and quantization for constrained systems," Physical Review E, vol. 84, no. 2, Article ID 026608, 2011.

[19] H. Kleinert, "Fractional quantum field theory, path integral, and stochastic differential equation for strongly interacting manyparticle systems," Europhysics Letters, vol. 100, no. 1, Article ID 10001, 2012. 
[20] D. Baleanu, "About fractional quantization and fractional variational principles," Communications in Nonlinear Science and Numerical Simulation, vol. 14, no. 6, pp. 2520-2523, 2009.

[21] E. Goldfain, "Derivation of lepton masses from the chaotic regime of the linear $\sigma$-model," Chaos, Solitons \& Fractals, vol. 14, no. 9, pp. 1331-1340, 2002.

[22] R. Herrmann, "Gauge invariance in fractional field theories," Physics Letters A, vol. 372, no. 34, pp. 5515-5522, 2008.

[23] R. Herrmann, “The fractional symmetric rigid rotor," Journal of Physics G: Nuclear and Particle Physics, vol. 34, no. 4, pp. 607625, 2007.

[24] G. Calcagni, "Fractal universe and quantum gravity," Physical Review Letters, vol. 104, no. 25, Article ID 251301, 2010.

[25] G. Calcagni, "Geometry of fractional spaces," Advances in Theoretical and Mathematical Physics, vol. 16, no. 2, pp. 549-644, 2012.

[26] K. Svozil and A. Zeilinger, "Dimension of space-time," International Journal of Modern Physics A, vol. 1, no. 4, pp. 971-990, 1986.

[27] G. Jumarie, "Table of some basic fractional calculus formulae derived from a modified Riemann-Liouville derivative for nondifferentiable functions," Applied Mathematics Letters, vol. 22, no. 3, pp. 378-385, 2009.

[28] F. H. Stillinger, "Axiomatic basis for spaces with noninteger dimension," Journal of Mathematical Physics, vol. 18, no. 6, pp. 1224-1234, 1976.

[29] A. Zeilinger and K. Svozil, "Measuring the dimension of spacetime," Physical Review Letters, vol. 54, no. 24, pp. 2553-2555, 1985.

[30] K. Svozil, "Quantum field theory on fractal spacetime: a new regularisation method," Journal of Physics A, vol. 20, no. 12, pp. 3861-3875, 1987.

[31] G. Eyink, "Quantum field-theory models on fractal spacetimeI. Introduction and overview," Communications in Mathematical Physics, vol. 125, no. 4, pp. 613-636, 1989.

[32] C. Palmer and P. N. Stavrinou, "Equations of motion in a noninteger-dimensional space," Journal of Physics A, vol. 37, no. 27, pp. 6987-7003, 2004.

[33] L. Nottale, "Scale relativity, fractal space-time and quantum mechanics," Chaos, Solitons \& Fractals, vol. 4, no. 3, pp. 361-388, 1994.

[34] P. Grigolini, A. Rocco, and B. J. West, "Fractional calculus as a macroscopic manifestation of randomness," Physical Review E, vol. 59, no. 3, pp. 2603-2613, 1999.

[35] G. Jumarie, "From Lagrangian mechanics fractal in space to space fractal Schrödinger's equation via fractional Taylor's series," Chaos, Solitons \& Fractals, vol. 41, no. 4, pp. 1590-1604, 2009.

[36] G. Jumarie, "Derivation and solutions of some fractional BlackScholes equations in coarse-grained space and time. Application to Merton's optimal portfolio," Computers and Mathematics with Applications, vol. 59, no. 3, pp. 1142-1164, 2010.

[37] G. Calcagni, A. Eichhorn, and F. Saueressig, "Probing the quantum nature of spacetime by diffusion," Physical Review D, vol. 87, Article ID 124028, 2013.

[38] G. Calcagni, "Geometry and field theory in multi-fractional spacetime," Journal of High Energy Physics, vol. 2012, no. 1, article 65, 2012.

[39] G. Calcagni, J. Magueijo, and D. R. Fernández, "Varying electric charge in multiscale spacetimes," Physical Review D, vol. 89, Article ID 024021, 2014.
[40] F. Riewe, "Nonconservative Lagrangian and Hamiltonian mechanics," Physical Review E, vol. 53, no. 2, pp. 1890-1899, 1996.

[41] F. Riewe, "Mechanics with fractional derivatives," Physical Review E, vol. 55, no. 3, pp. 3581-3592, 1997.

[42] C. F. L. Godinho, J. Weberszpil, and J. A. Helayël-Neto, "Fractional canonical quantization: a parallel with noncommutativity," International Journal of Theoretical Physics, vol. 51, pp. 1-17, 2014.

[43] A. O. Caldeira and A. J. Leggett, "Influence of dissipation on quantum tunneling in macroscopic systems," Physical Review Letters, vol. 46, no. 4, pp. 211-214, 1981.

[44] A. O. Caldeira and A. J. Leggett, "Quantum tunnelling in a dissipative system," Annals of Physics, vol. 149, no. 2, pp. 374456, 1983.

[45] J. Ankerhold, H. Grabert, and G.-L. Ingold, "Dissipative quantum systems with a potential barrier: general theory and the parabolic barrier," Physical Review E, vol. 51, no. 5, pp. 42674281, 1995.

[46] I. R. Senitzky, "Dissipation in quantum mechanics. the harmonic oscillator," Physical Review, vol. 119, no. 2, pp. 670-679, 1960.

[47] K. W. H. Stevens, "The wave mechanical damped harmonic oscillator," Proceedings of the Physical Society, vol. 72, no. 6, pp. 1027-1036, 1958.

[48] A. K. Golmankhaneh, "Relativistic scalar fields for nonconservative systems," Physica Scripta, vol. 2009, Article ID 014008, 2009.

[49] M. J. Lazo, "Fractional variational problems depending on fractional derivatives of differentiable functions with application to nonlinear chaotic systems," Conference Papers in Mathematics, vol. 2013, Article ID 872869, 5 pages, 2013.

[50] G. M. Zaslavsky, Hamiltonian Chaos and Fractional Dynamics, Oxford University Press, Oxford, UK, 2005.

[51] C. F. L. Godinho, J. Weberszpil, and J. A. Helayël-Neto, "Extending the D'alembert solution to space-time Modified RiemannLiouville fractional wave equations," Chaos, Solitons \& Fractals, vol. 45, no. 6, pp. 765-771, 2012.

[52] M. S. Plyushchay and M. Rausch de Traubenberg, "Cubic root of Klein-Gordon equation," Physics Letters B, vol. 477, no. 1-3, pp. 276-284, 2000.

[53] A. Raspini, "Simple solutions of the fractional Dirac equation of order 2/3," Physica Scripta, vol. 64, no. 1, pp. 20-22, 2001.

[54] P. Zavada, "Relativistic wave equations with fractional derivatives and pseudodifferential operators," Journal of Applied Mathematics, vol. 2, no. 4, pp. 163-197, 2002.

[55] I. Podlubny, Fractional Differential Equations, vol. 198 of Mathematics in Science and Engineering, Academic Press, San Diego, Calif, USA, 1999.

[56] A. A. Kilbas, H. M. Srivastava, and J. J. Trujillo, Theory and Applications of Fractional Differential Equations, vol. 204 of North-Holland Mathematics Studies, Elsevier Science B.V., Amsterdam, The Netherlands, 2006.

[57] S. G. Samko, A. A. Kilbas, and O. I. Marichev, Fractional Integrals and Derivatives, Gordon and Breach, New York, NY, USA, 1993.

[58] K. S. Miller and B. Ross, An Introduction to the Fractional Calculus and Fractional Differential Equations, A Wiley-Interscience Publication, John Wiley \& Sons, New York, NY, USA, 1993.

[59] B. Ross, Ed., Fractional Calculus and Its Applications, vol. 457 of Lecture Notes in Mathematics, Springer, New York, NY, USA, 1975. 
[60] G. Jumarie, "An approach to differential geometry of fractional order via modified Riemann-Liouville derivative," Acta Mathematica Sinica, vol. 28, no. 9, pp. 1741-1768, 2012.

[61] G. Jumarie, "On the derivative chain-rules in fractional calculus via fractional difference and their application to systems modelling," Central European Journal of Physics, vol. 11, no. 6, pp. 617-633, 2013.

[62] L. Longjin, F.-Y. Ren, and W.-Y. Qiu, "The application of fractional derivatives in stochastic models driven by fractional Brownian motion," Physica A, vol. 389, no. 21, pp. 4809-4818, 2010.

[63] J. Weberszpil and J. J. Ramón-Marí, to be submitted, 2014.

[64] G. Jumarie, "White noise calculus, stochastic calculus, coarsegraining and fractal geodesic. A unified approach via fractional calculus and Maruyamas notation," in Brownian Motion: Theory, Modelling and Application, R. C. Earnshaw and E. M. Riley, Eds., pp. 1-69, Nova Publishing, 2011.

[65] K. M. Kolwankar and A. D. Gangal, "Local fractional calculus: a calculus for fractal space-time," in Fractals: Theory and Applications in Engineering, pp. 171-181, Springer, London, UK, 1999.

[66] K. M. Kolwankar and A. D. Gangal, "Fractional differentiability of nowhere differentiable functions and dimensions," Chaos, vol. 6, no. 4, pp. 505-513, 1996.

[67] K. M. Kolwankar and A. D. Gangal, "Local fractional FokkerPlanck equation," Physical Review Letters, vol. 80, no. 2, pp. 214217, 1998.

[68] A. Babakhani and V. Daftardar-Gejji, "On calculus of local fractional derivatives," Journal of Mathematical Analysis and Applications, vol. 270, no. 1, pp. 66-79, 2002.

[69] Y. Chen, Y. Yan, and K. Zhang, "On the local fractional derivative," Journal of Mathematical Analysis and Applications, vol. 362, no. 1, pp. 17-33, 2010.

[70] A. Carpinteri and A. Sapora, "Diffusion problems in fractal media defined on Cantor sets," Zeitschrift für Angewandte Mathematik und Mechanik, vol. 90, no. 3, pp. 203-210, 2010.

[71] W. Chen, "Time-space fabric underlying anomalous diffusion," Chaos, Solitons \& Fractals, vol. 28, pp. 923-929, 2006.

[72] W. Chen, H. Sun, X. Zhang, and D. Korošak, "Anomalous diffusion modeling by fractal and fractional derivatives," Computers and Mathematics with Applications, vol. 59, no. 5, pp. 1754-1758, 2010.

[73] V. Kobelev, "The variant of post-Newtonian mechanics with generalized fractional derivatives," Chaos, vol. 16, Article ID 043117, 2006.

[74] A. A. Budini and M. O. Cáceres, "Functional characterization of generalized Langevin equations," Journal of Physics A, vol. 37, no. 23, pp. 5959-5981, 2004.

[75] M. Bologna, P. Grigolini, and J. Riccardi, "Lévy diffusion as an effect of sporadic randomness," Physical Review E, vol. 60, no. 6A, pp. 6435-6442, 1999.

[76] R. Herrmann, "Common aspects of q-deformed Lie algebras and fractional calculus," Physica A, vol. 389, no. 21, pp. 4613$4622,2010$.

[77] J. Weberszpil, C. F. L. Godinho, A. Cherman, and J. A. HelayëlNeto, "Aspects of the coarse-grained-based approach to a lowrelativistic fractional Schrödinger equation," in Proceedings of the 7th Conference Mathematical Methods in Physics (ICMP '12), pp. 1-19, Proceedings of Science (PoS), Rio de Janeiro, Brazil, 2012.
[78] S. Eidelmana, M. Giacominib, F. V. Ignatova, and M. Passera, "The $\tau$ lepton anomalous magnetic moment," Nuclear Physics B-Proceedings Supplements, vol. 169, pp. 226-231, 2007. 

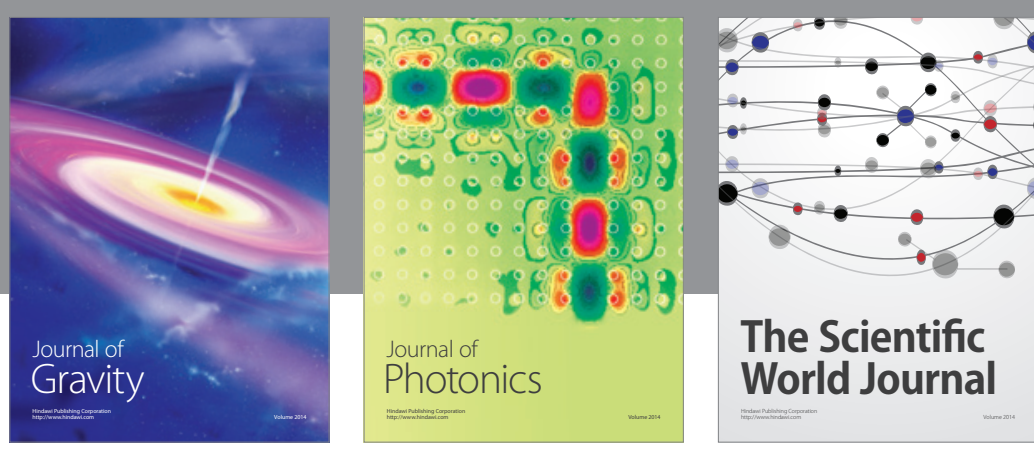

The Scientific World Journal
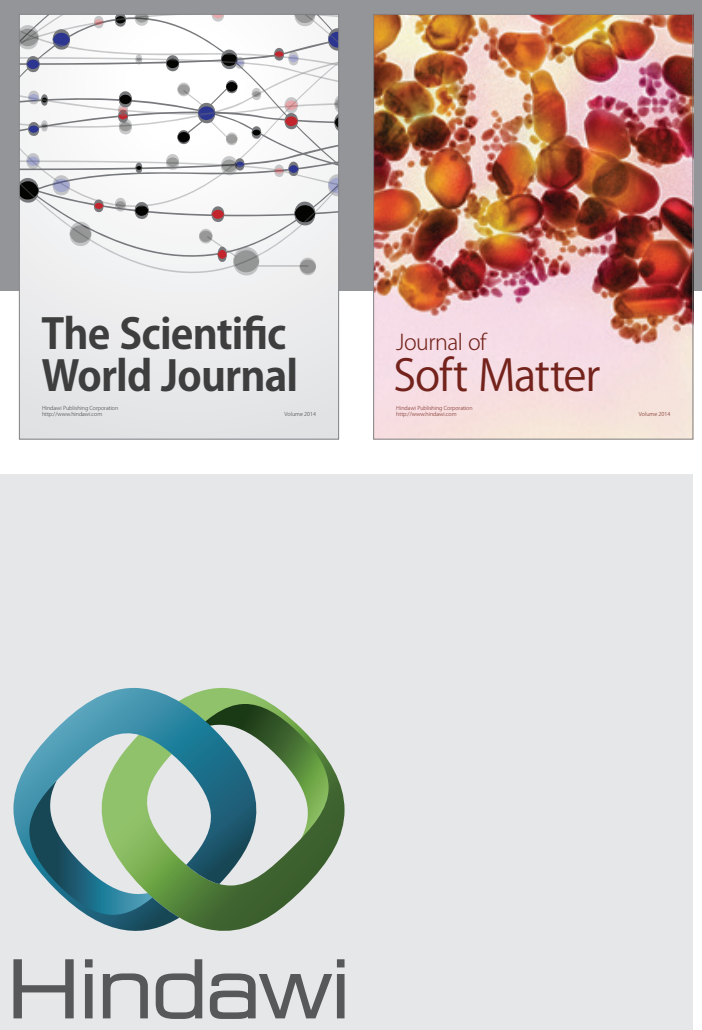

Submit your manuscripts at

http://www.hindawi.com

nternational Journal of

Statistical Mechanics
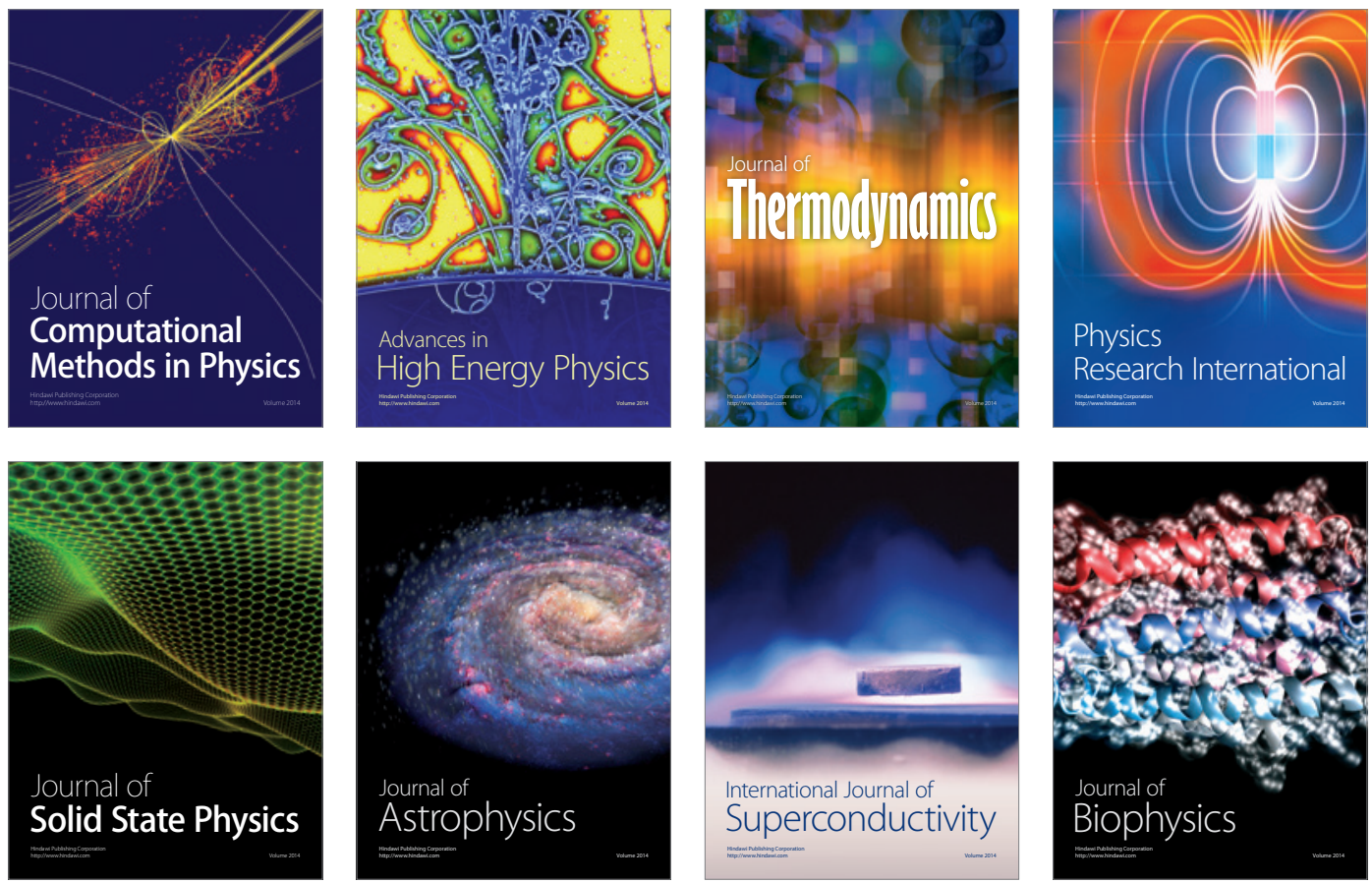
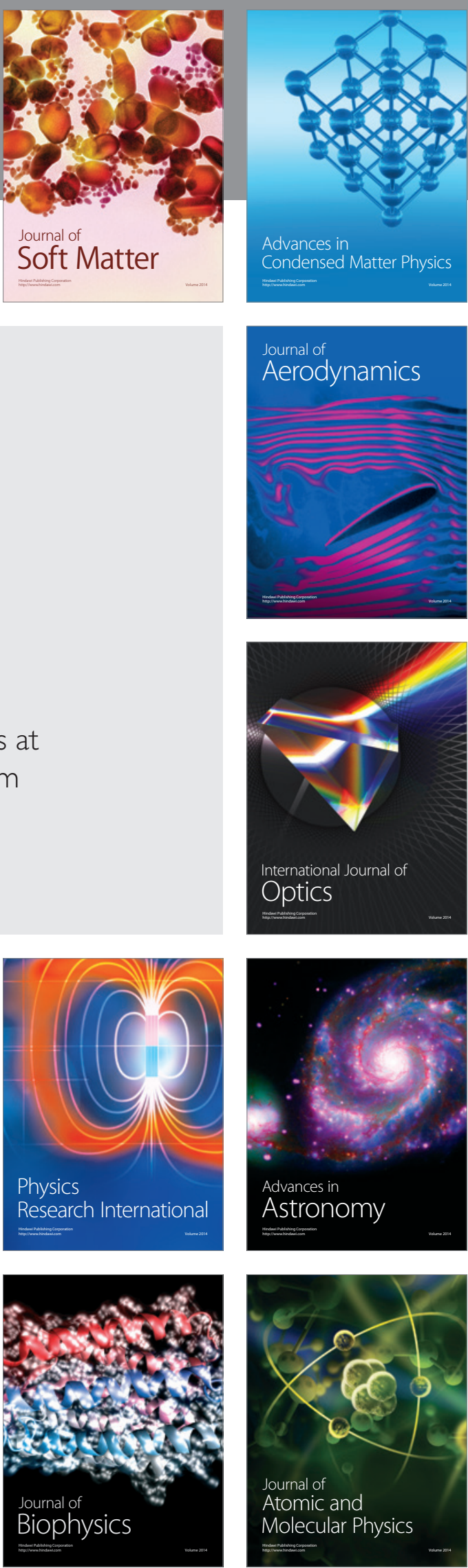